\title{
Catastrophic hemothorax on the contralateral side of the insertion of an implantable subclavian venous access device and the ipsilateral side of the removal of the infected port
} -A case report-

\author{
Ji-Heui Lee, Young Bae Kim, Min Kee Lee, Jong Il Kim, Ji-Yeon Lee, So Young Lee, Eun-Ju Lee, and \\ Yong Seock Lee
}

Department of Anesthesiology and Pain Medicine, Korean Institute of Radiological \& Medical Sciences, Seoul, Korea

The percutaneous placement of a totally implantable vascular access port via the subclavian vein is commonly used in patients with a need for multiple intravenous infusions such as administration of chemotherapy. Unfortunately, the use of central venous ports have been associated with adverse events that are hazardous to patients. Here we report the case of a 5-year-old child who died of catastrophic hemothorax after several attempts at insertion of an implantable subclavian venous access device and removal of an infected port. Massive hemothorax occurred on the side contralateral to unsuccessful attempts at insertion of a new port and ipsilateral to the removal of an infected port. We could not confirm the cause of death and hemothorax without autopsy, but we discuss several possible causes of massive hemothorax. (Korean J Anesthesiol 2010; 59: 214-219)

Key Words: Cause of death, Hemothorax, Subclavian vein, Vascular access port.

The use of totally implantable venous access devices (TICVAD) have greatly resolved and simplified the administration of chemotherapy and the sampling of blood in patients with malignancies. Since Hickman's original description in 1979 [1], the use of implantable central venous access devices has increased. Long-term central venous access has become vital in the management of children with gastrointestinal malformation, hematopoietic disorders, and cancer. Therefore, the prevalence of anesthesia usage in the implantation and removal of TICVAD have increased in pediatric thoracic surgeries and other pediatric surgeries.

Numerous major complications with TICVAD and percutaneous central venous catheterization have been reported, but complications that occur after the removal of the central venous catheter have only rarely been reported. For example, we found no reports on the occurrence of hemothorax after the removal of a central venous catheter on the opposite side of the central venous catheterization. Therefore, we present the current case

Received: February 12, 2010. Revised: 1st, March 2, 2010; 2nd, March 10, 2010. Accepted: March 10, 2010.

Corresponding author: Ji-Heui Lee, M.D., Department of Anesthesiology and Pain Medicine, Korean Institute of Radiological \& Medical Sciences, Gongneung-dong, Nowon-gu, Seoul 139-240, Korea. Tel: 82-2-970-2820, Fax: 82-2-970-2413, E-mail: jiheui0255@naver.com (c) This is an open-access article distributed under the terms of the Creative Commons Attribution Non-Commercial License (http:// creativecommons.org/licenses/by-nc/3.0/), which permits unrestricted non-commercial use, distribution, and reproduction in any medium, provided the original work is properly cited. 
report of a pediatric patient who died of right catastrophic hemothorax that occurred after the removal of an infectious right TICVAD and an attempt to implant a left TICVAD.

\section{Case Report}

A 5-year-old male patient of $111 \mathrm{~cm}$ and $19 \mathrm{~kg}$, who experienced an osteosarcoma in the left fibula was to go undergo insertion of a totally implantable central venous access device for chemotherapy. But the right totally implantable subclavian central venous catheter was infected. Hence, the patient was admitted for surgery for the removal of the infected catheter and for left totally implantable subclavian central venous catheterization. The patient underwent numerous chemotherapy treatments (CDDP, Adriamycin, Methotrexate, Ifofamide, VP-16). He had been on antibiotics for over 3 weeks due to an infection at the TICVAD site.

In the blood test before surgery, the platelet level had decreased to $64,000 / \mathrm{mm}^{3}$. Electrocardiography, echocardiography, and chest x-ray tests appeared normal. While the patient was on the OR table, a very severe infectious ulcer and abscess of $5 \mathrm{~cm}$ in diameter was observable to the human eye on the skin and the tissue below the skin at the right TICVAD site.

No premedication was given. On the operating table, the EKG, pulse oximeter, and a noninvasive blood pressure cuff were attached. The measured systolic/diastolic blood pressure was 100/50 mmHg, and heart rate was 90 . Glycopyrrolate (0.06 mg) and thiopental (100 mg) were administered to induce loss of consciousness. Oxygen and sevoflurane were used for manual ventilation. After securing airway patency, fentanyl $20 \mu \mathrm{g}$ and rocuronium $15 \mathrm{mg}$ were administered. After adequate muscle relaxation, a noncuffed endotracheal tube (I.D. $5.0 \mathrm{~mm}$ ) was inserted. After endotracheal intubation, $\mathrm{N}_{2} \mathrm{O}$ and oxygen were used to maintain the inspired oxygenconcentration at 0.5 . Sevoflurane $1.5-3$ vol\% was used for anesthesia during manual ventilation. For the end-tidal $\mathrm{CO}_{2}$ concentration to be $35 \mathrm{mmHg}$, the tidal volume and the respiratory rate were controlled. The thoracic surgeon used a $21 \mathrm{G}$. introducer needle to puncture the left subclavian vein. For this procedure, we reduced the manual ventilation to prevent the occurrence of pneumothorax. When the left vessel was punctured, the beat of the arterial blood was not found in the small amount of aspired blood. So the blood was assumed to be venous. While inserting a guide wire and a 6.5 Fr. dilator, aspiration of blood that appeared to be arterial blood was observed. Therefore, all catheters were quickly removed, and pressure was applied for hemostasis. Afterwards, puncture of the left subclavian vein was attempted again, but the introducer needle was removed after repeated arterial puncture, and pressure was applied for hemostasis. Venous puncture was attempted 2 or 3 times, but it failed. Then puncture of the left internal jugular vein was attempted, but it also failed.

A slight edema was noted in the neck and the left subclavian area. So the puncture attempts on the upper body were stopped. Instead, the puncture of the left femoral vein was attempted successfully. A 6 Fr. TICVAD (X-Port ${ }^{\circledR}$ Bard Access Systems, USA) was inserted. During the venous puncture, the patient was assumed to have hemorrhaged. So a plasma expander (HESplazma ${ }^{\circledR} 6 \%$, ChoongWae, Korea) $200 \mathrm{ml}$ was infused. During the central venous catheterization, no serious decrease in blood pressure was found. The right central venous catheter and the port were removed.

Curettage of the tissue near the infection was performed, and then the site was closed, ending the surgery. After the surgery, the patient was completely awake, could open his eyes, and move his arms and legs. However, a sudden drop in blood pressure occurred. The arterial hematocrit was measured at $20 \%$. A vasopressor, ephedrine $2.5 \mathrm{mg}$ and packed RBC $1 \mathrm{U}$ were administered. Then the arterial hematocrit rose to $28 \%$. Because of a continuous drop in blood pressure (60-90/30$40 \mathrm{mmHg}$ ) and acute acidemia, dopamine $\mathrm{HCl}$ (Dopamine free mix $80 \mathrm{mg}^{\circledR}, \mathrm{CJ}$, Korea $10-20 \mu \mathrm{g} / \mathrm{kg} / \mathrm{min}$ ) was continuously administered, and bicarbonate was intermittently administered. Then midazolam (1 mg)and rocuronium (5 mg) were administered. In the first chest x-ray (Fig. 1), a very large right hemothorax was discovered. Immediately, the thoracic surgeon inserted a chest tube and $500 \mathrm{ml}$ of blood was drained. Packed RBC were continuously administered, which helped maintain the arterial hematocrit level at 33-35\%. Continuous low blood pressure (60-90/ 30-40 mmHg) and tachycardia (140-150 bpm) were observed. Hence, dopamine

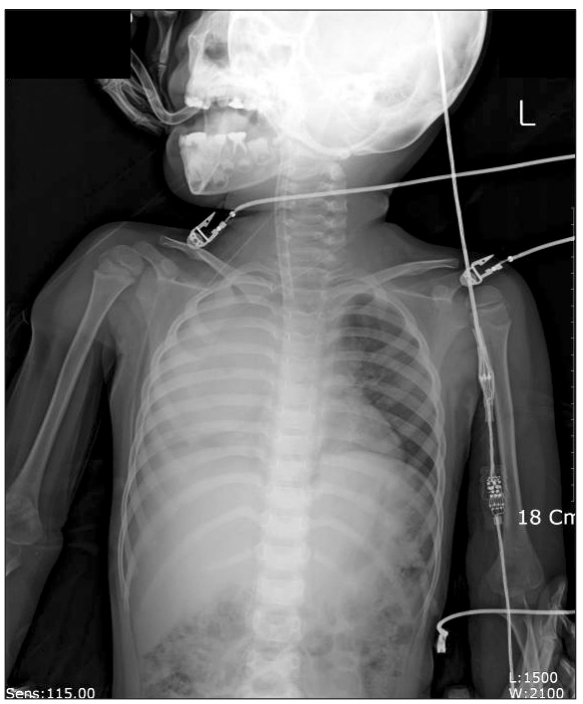

Fig. 1. Chest radiograph after surgery shows a large hematoma in the right thoracic cavity. 
was continuously administered. Acidemia $(\mathrm{pH}=7.051$, Base Excess $=-16 \mathrm{mEq} / \mathrm{L})$ and hyperkalemia $(\mathrm{K}+=6.2 \mathrm{mmol} / \mathrm{L})$ were found, so bicarbonate, calcium gluconate, and diuretics were intermittently administered. Midazolam and vecuronium were used for maintaining anesthesia. When 3U of Packed $\mathrm{RBC}$ were administered, the airway pressure started to slowly increase. When $24-26 \mathrm{~cm} \mathrm{H}_{2} \mathrm{O}$ was measured, the tidal volume was decreased and the respiratory rate was increased. In a retaken chest x-ray (Fig. 2), a large amount of blood had not been drained from the right chest area, so a second tube was inserted in the chest. Afterwards, over 2,000 ml of blood was

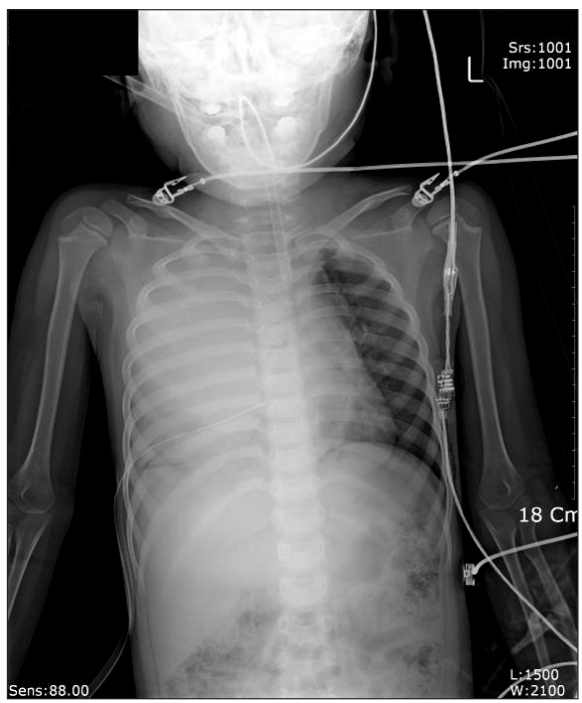

Fig. 2. Chest radiograph after the insertion of the 1st chest tube. Massive hematoma was observed in the right thorax.

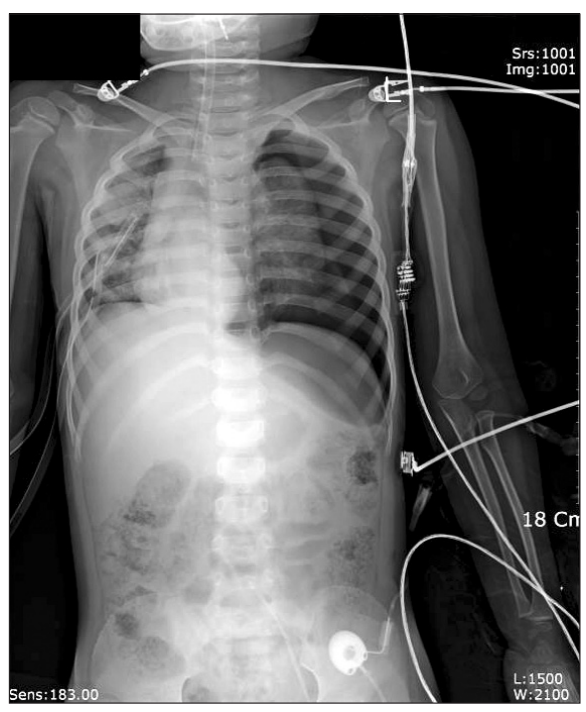

Fig. 3. Chest radiograph after insertion of the 2nd chest tube. Hematoma in the right thoracic cavity was reduced but left pneumothorax appeared. drained. Using a stethoscope, we found that the left pulmonary sound was reduced. Hence, in the next chest x-ray (Fig. 3), the right hemothorax was almost all gone, but a pneumothorax on the left-side was discovered. Thus, a third chest tube was inserted. However, the first and second chest tubes on the right-side continuously drained blood, and the blood pressure was not maintainable at $40-70 \mathrm{mmHg} / 20-40 \mathrm{mmHg}$. So, after discussions with the thoracic surgeon, a median sternotomy was done for hemostasis. A mid-surgery increase in hemorrhaging was predicted, so an $18 \mathrm{G}$ intravenous catheter was inserted into the right external jugular vein. The hematocrit level in the arterial blood was maintained at $33-37 \%$. The drop in blood pressure could not be corrected, so a large amount of epinephrine $0.5-1.0 \mu \mathrm{g} / \mathrm{kg} / \mathrm{min}$ was continuously administered. Epinephrine $(10-100 \mu \mathrm{g})$, bicarbonate, calcium gluconate, and diuretics were intermittently administered. In the sternotomy, damage to the main blood vessels (the subclavian vein, the subclavian artery, and the pulmonary artery and vein) was not discovered. However, a severe bulging of the parietal pleura in the upper portion of the right chest, a dissection between the parietal pleura and visceral pleura, and a hematoma between the 2 pleurae were observed (Fig. 4). In the posterior portion of the right upper thorax, there was an abscess pocket, which burst and leaked abscess in midsurgery. The patient was assumed to be in a septic state, so the pediatrician administered dexamethazone $5 \mathrm{mg}$. However, the patient continued to have low blood pressure. The thoracic surgeon could not find the exact hemorrhage site on the right-

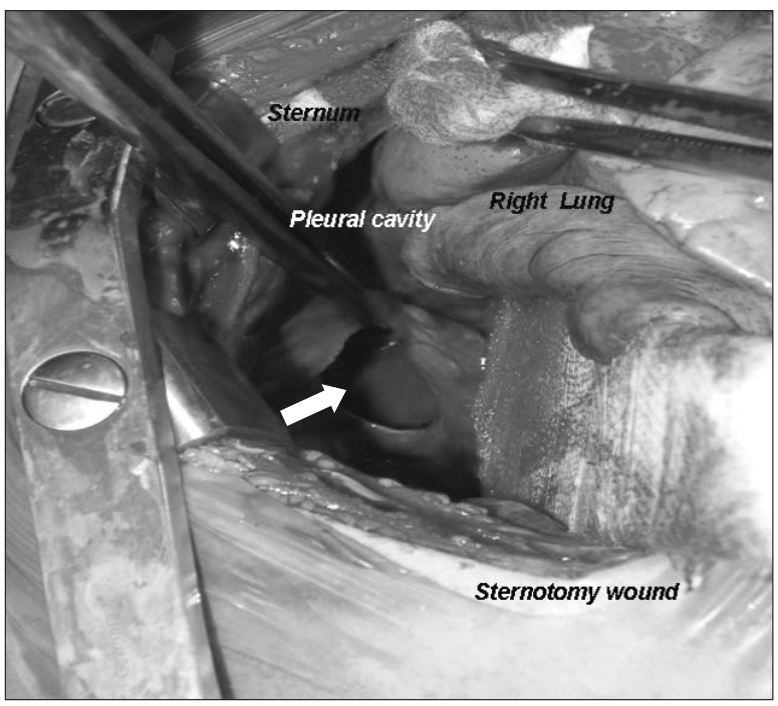

Fig. 4. The patient underwent a midline sternotomy, at which time dissected space between parietal, visceral pleura and chest wall are filled with blood and a hematoma (white arrow). Blood and the hematoma are evacuated after making an incision in the bulging pleura. 
side of the chest. Because of the progression of the disseminated coagulation disorder, it was deemed not viable to continue with the surgery, so the surgery was ended. While closing up the patient's chest, $900 \mathrm{cc}$ of blood was drained from the chest tube. Packed RBC and fresh frozen plasma $3 \mathrm{U}$ were administered. A continuous high concentration of epinephrine and dopamine, intermittent epinephrine, calcium gluconate, and bicarbonate were administered. In the OR, the administered amount of fluid and blood amounted to Packed RBC $10 \mathrm{U}$, fresh frozen plasma $3 \mathrm{U}$, and fluid 3,000 $\mathrm{ml}$. After surgery, the patient was moved to the ICU and connected to an EKG when bradycardia was observed. Atropine and epinephrine were administered, and blood pressure was reduced $(60 / 30 \mathrm{mmHg})$. In the ICU, blood continued to drain from the chest tube. So Packed RBC were continuously administered, but the hematocrit level was $24 \%$. Within 20 minutes of arriving at the ICU, the patient experienced cardiac arrest. Thus, cardiopulmonary resuscitation was immediately performed. A cardiac beat and a measurable blood pressure were found. However, 15 minutes later, cardiac arrest was again observed, so cardiopulmonary resuscitation was attempted. The patient died 1 hour after the end of surgery.

\section{Discussion}

The use of a totally implantable central venous access port provides long-term central venous access and thus simplifies the administration of chemotherapy, parenteral nutrition, and the repeated collection of blood samples. In contrast to the percutaneous central venous catheterization, dilators and peel-away sheaths are used to insert the catheter. Two small skin incisions are required: one at the guide wire exit site and a second for the implantation of the port reservoir $(4 \mathrm{~cm})$. In most cases, catheterization is done in the subclavian vein, but it can be done in the internal jugular vein, the external jugular vein, and the common femoral vein.

During subclavian venous catheterization, arterial punctures occur $6-8 \%$ of the time [2]. For pediatric patients, the rate is $10 \%$ [3], but this has not been studied much. Hemorrhage usually occurs as a result of the puncture of the subclavian artery, the pulmonary artery and other adjacent vessels such as the intercostal artery and the internal mammary artery [4]. Moreover, such hemorrhages are easily concealed due to blood tracking in the pleural cavity. The artery is in an anatomically non-compressible location, which means that local pressure is ineffectual. In the present case, the thoracic surgeon punctured the subclavian artery twice. The first time, a vessel dilator was also inserted, which may have caused damage to the left subclavian artery and hemorrhage and triggered the hemothorax. However, this cannot explain why repeated $\mathrm{x}$-rays showed no hemorrhage at all in the left chest-area and only found that the opposite side had a massive hemothorax.

Hemothorax occurring from a subclavian venous cannulation can cause an acute loss of blood and circulatory collapse. In such cases, with the use of an angiogram, stent implantation or surgical treatment is required. But then it becomes difficult to reach the hemorrhage site of the thoracic apex [5]. In the case of our pediatric patient, the first chest tube was inserted in the lower part of the right thorax. We could not drain the hematoma. So, a chest tube was inserted into the upper portion of the thorax and 2,000 ml of blood was drained. The amount of hemorrhaging did not decrease, so a median sternotomy was considered for hemostasis. However, a large amount of hemorrhaging and transfusion had caused a disseminated coagulation dysfunction and a platelet reduction. So surgery alone did not suffice for hemostasis. A definite diagnosis of right-side hemorrhage could not be made, so both sides of the thorax were checked during the median sternotomy.

The cause of the hemothorax in our patient could not be found without an autopsy. Nevertheless, the authors attempted to find the cause by considering the events before and after the occurrence of the hemothorax and by weighing the information from other reported cases.

Hemothorax is a common sequela of chest trauma, an underlying malignancy, a ruptured aortic aneurysm, and inadvertent vessel injury in a central venous cannulation or a tube thoracostomy [6]. When hemothorax is noted without any obvious trauma, it is called spontaneous hemothorax or nontraumatic hemothorax. Causes implicated in the etiology of the spontaneous hemothorax include neoplasms, coagulation disorders, AV malformations, ruptured aneurysms, endometriosis, and pulmonary vasculature malformations [6]. The pseudoaneurysm can be caused by central venous cannulation. A few cases of pseudoaneurysm of the subclavian artery, the internal mammary artery, the intercostal artery, the brachiocephalic trunk, the thyrocervical trunk and the innominate artery following subclavian vein catheterization attempts have been reported as the cause of spontaneous hemothorax $[7,8]$. Other causes include traumas, infections, vasculitis, and tumors. Some have reported internal mammary artery pseudoaneurysms that complicated severe chest wall infection in children [9]. Therefore, anterior chest wall abscesses should be treated aggressively to prevent the transthoracic spread of an infection leading to vascular complications. In the present case, our patient had the subclavian venous port removed. Within a few minutes, a severe drop in blood pressure was noted. The sternotomy showed that the abscess had spread into the thorax. An inflammatory ulcer and an abscess at the insertion point of the port may have caused the vascular abnormalities in the thorax. Or the catheter itself may have 
caused mechanical irritation causing vascular abnormalities in the area. Also, in the process of removing the catheter, the rupture of the vascular abnormalities may have caused the hemothorax. During the sternotomy, no abnormalities could be found in the subclavian vein and artery. Pseudoaneurysms in adjacent nearby vessels, such as the intercostal artery, the intermammary artery, or the brachiocephalic trunk could have ruptured and caused the hemothorax.

The formation of pseudoaneurysms can be diagnosed on contrast angiography, contrast computed tomography, and pulmonary arterial angiography. Methods of treatment are endoscopic coagulation, angiographic embolization, surgical resection, or repair [7-9]. Our patient did not have an angiography or a CT scan taken. Pseudoaneurysm cannot be discovered from a simple thoracic $\mathrm{x}$-ray, so a definite diagnosis could not be made. The median sternotomy showed the right upper pleural apex drooping to the lower part, where the acute infection was seen. We assume the formation of pseudoaneurysm in this area.

A hemothorax of unknown cause is called an idiopathic spontaneous hemothorax. They are usually accompanied by pneumothorax. Kim et al. [10] reported that spontaneous hemothorax is caused by a small vascular rupture of adhesions between the visceral pleura and parietal pleura, which is caused by pulmonary collapse from damage to vascular pulmonary bullae or other factors. Bleeding adhesions are identified as the cause of hemothorax in up to $53 \%$ cases of spontaneous hemopneumothorax. The adhesions are commonly found near the apex and can also be found near the aorta, the subclavian vessels, and the pericardium. Explanations for the substantial bleeding from these torn adhesions include absence of a tamponade effect, lung movement impeding clot formation, and defective muscle components in the vessel wall. For hemostasis in a pediatric patient, median sternotomy was performed, which led to us finding a severe bulging of the parietal pleura in the upper portion of the right chest, dissection between the parietal pleura and visceral pleura, and a large hematoma between the 2 pleurae. Considering that pneumothorax occurred on the left side and hemothorax on the right, it cannot be diagnosed as spontaneous hemopneumothorax. The cause of the pneumothorax on the opposite side may be from pulmonary penetration by the introducer needle or dilator. Or it could be from barotrauma caused by continuous elevation of air pressure due to the hemothorax or the infusion of a large amount of blood and fluid. Many authors have reported on ipsilateral hemothorax from a central venous catheterization $[4,5,7,8]$. However, hemothorax on the opposite side has rarely been reported $(0.4-0.7 \%)[11,12]$. One case was reported where, during placement, the subclavian vein, the pleura, and the pulmonary tissue were punctured and the result was contralateral tension pneumo- and hemo-thorax [11]. One case of acute contralateral hemothorax due to the puncture of the superior vena cava during the left subclavian vein cannulation for hemodialysis was reported [12]. In the present case, when the central venous catheter was inserted on the left, the deep insertion of the dilator could have caused damage to either the subclavian vein or other vessels and caused a large hemothorax. However, the thoracic surgeon discovered during the sternotomy that there was no significant damage to the vessels.

The reports of complications after the removal of a central venous catheter are rare, and hemothorax after the removal of central venous catheter is also very rare. A case of massive hemothorax developing a few minutes after the removal of a central venous catheter was reported in a patient who had an arteriovenous fistula for hemodialysis on the same side as the arm arteriovenous fistula [13]. The investigators recommended avoiding positioning the central catheter on the same side as an arm arteriovenous fistula. They emphasized that it was necessary to monitor patients after the removal of the subclavian vein catheter to detect this potentially fatal complication. Some postulated that it was likely that damage to the arterial adventitia had occurred during catheter insertion or use, which was not apparent as long as the catheter had remained in situ because of the "tamponading" effect by the catheter on the damaged artery [14].

Only upon removal of the catheter and release of the "tamponade" does the integrity of the arterial wall fail, resulting in formation of an aneurysm. Such damage to the adventitia is not apparent at the time of the implantation because the arterial lumen is not entered. It can occur either from grazing of the artery by the implantation needle or guide wire, or from rubbing of the catheter on the arterial adventitia during its use. To date, reports on hemothorax are rare when a central venous catheter is removed from a child. We found just one precedent, a 2 year old male patient who had a serious hemothorax when the central venous catheter was removed from the right jugular vein [15]. Complications with central venous cannulation must be looked for, and a routine chest x-ray must be performed. It is interesting that complications after the removal of central venous catheters are infrequently, if ever reported. This may be partly because such complications are not so actively looked for.

When fusing medical knowledge of reported cases with the physical condition of our patient, we came up with the following list of possible causes of the hemothorax. (1) A complication from the left subclavian venous catheter puncture. In other words, when the left subclavian vein was punctured, the right subclavian vein or other blood vessels could have been damaged. However, there was no damage of the right vessels 
found in the sternotomy, so this possibility is not likely. (2) The hemothorax might have occurred from a torn vessel located in the dissection between the right parietal and visceral pleurae. The dissection of the pleurae may have occurred spontaneously or from catheterization. (3) The hemothorax could have been caused after the removal of the infected right subclavian venous catheter. Our patient's blood platelet count could have decreased from the cancer treatment. A great amount of blood may have flown from the subclavian vein where the subclavian venous catheter was removed. But we did not find serious hemorrhaging near the right subclavian vein during the sternotomy. (4) Given that we found a lot of blood between right parietal pleura and viscerial pleura during the sternotomy, the parietal pleura drooped to a lower portion, and an abscess pocket developed in the right thorax, it is possible that a pseudoaneurysm near the blood vessels could have formed. In the process of removing the infected subclavian venous catheter, drainage near the pseudoaneurysm could have caused heavy hemorrhage. In many reports, the most likely possibility of vascular findings are intercostal arterial- and internal mammary pseudoaneurysms. However, during the sternotomy, the pseudoaneurysm had already ruptured. Because the patient experienced septicemia and disseminated coagulation dysfunction, his thoracic region experienced hemorrhaging. Therefore, the exact location of the hemorrhage could not be found. So this possibility cannot be confirmed. Nevertheless, since a simple surgery such as a TICVAD catheterization can lead to critical conditions or even death, we emphasize the dangers and complications of central venous catheterization in the field of anesthesia. Hence, we emphasize the great value patient observations. In both the insertion and the removal of the catheter, serious complications can occur. When an infected central venous catheter is removed, because the adjacent blood vessels can have complications, careful patient observation is always required.

\section{References}

1. Hickman RO, Buckner RAC, Sanders JE, Stewart P, Thomas ED. A modified right atrial catheter for access to the venous system in marrow transplant recipients. Surg Gynecol Obstet 1979; 148: 871-5.

2. Ruesch B, Walder B, Tramer MR. Complications of central venous catheters: internal jugular versus subclavian access: a systematic review. Crit Care Med 2002; 30: 454-60.

3. Citak A, Karabocuoglu M, Ucsel R, Uzel N. Central venous catheters in pediatric patients-subclavian venous approach as the first choice. Pediatr Int 2002; 44: 83-6.

4. Lee CS, Kim JT, Kim CS, Kim SD, Kim HS. Hemothorax due to injuries of subclavian artery and first intercostal artery after subclavian venous catheterization in a pediatric patient. Korean J Anesthesiol 2009; 56: 587-91.

5. Nicholson T, Ettles D, Robinson G. Managing inadvertent arterial catheterization during central venous access procedures. Cardiovasc Intervent Radiol 2004; 27: 21-5.

6. Berliner K. Hemorrhagic pleural effusion. An analysis of 120 cases. Ann Intern Med 1941; 14: 2266-84.

7. Koklu E, Poyrazoglu H, Vikilmaz A, Campolat M, Konuskan B. Subclavian artery psudoaneurysm: a rare and serious complication of central venous catheterization in an infant. Pediatr Radiol 2008; 38: 220-3.

8. Kruyt PM, Winter LH, Koning J. A pseudo-aneurysm of the internal mammary artery: a very rare complication of subclavian vein puncture. Eur J Vasc Surg 1993; 7: 349-51.

9. Deshmukh H, Prasad SR, Patankar T, Zankar M. Internal mammary artery pseudoaneurysms complicating chest wall infection in children diagnosis and endovascular therapy. Clin Imaging 2001; 25: 396-9.

10. Kim ES, Kang JY, Pyo CH, Jeona EY, Lee WB. 12-year experience of spontaneous hemopneumothorax. Ann Thorac Cardiovasc Surg 2008; 14: 149-53.

11. Lock RL, Triplett HB, Rse G. Contralateral tension pneumo/hemothorax resulting from left subclavian vein cannulation under general anesthesia. Nurse Anesth 1991; 2: 89-92.

12. Rodriguez J, Barcena M, Alvarez J. Acute contralateral hemothorax after cannulation of the left subclavian vein for hemodialysis. Rev Esp Anestesiol Reanim 2002; 49: 428-31.

13. Andrea C, Stefano N, Giulianan R, Mario C. Massive hemothorax after removal of subclavian vein catheter: a very unusual complication. Crit Care Med 2002; 30: 697-8.

14. Walden FM. Subclavian aneurysm causing brachial plexus injury after removal of a subclavian catheter. Br J Anaesth 1997; 79: 807-9.

15. Iwakura H, Hashimoto K, Nomura T, Morimoto N, Sito Y, Kosaka Y. A malpositioned CVP catheter. Masui 1997; 46: 1374-7. 